\title{
Linx
}

Revue des linguistes de l'université Paris X Nanterre

49 | 2003

L'actualité des notions d'interlangue et d'interaction exolingue

\section{En CLIN, l'apprentissage du français passe par la socialisation en français}

Marie-Thérèse Vasseur

\section{CpenEdition}

Journals

Édition électronique

URL : http://journals.openedition.org/linx/560

DOI : $10.4000 / \operatorname{lin} x .560$

ISSN : 2118-9692

Éditeur

Presses universitaires de Paris Nanterre

Édition imprimée

Date de publication : 1 décembre 2003

Pagination : 125-140

ISSN : 0246-8743

Référence électronique

Marie-Thérèse Vasseur, «En CLIN, l'apprentissage du français passe par la socialisation en français », Linx [En ligne], 49 | 2003, mis en ligne le 18 mars 2011, consulté le 19 avril 2019. URL : http:// journals.openedition.org/linx/560 ; DOI : 10.4000/linx.560 


\title{
En CLIN, l'apprentissage du français passe par la socialisation en français
}

\author{
Marie-Thérèse Vasseur \\ UMR 8606, CNRS et Université du Maine
}

L'acquisition d'une langue étrangère a longtemps été étudiée du strict point de vue du développement de la langue. Cette approche, si elle permet de suivre, à partir du repérage des formes, le développement de la langue de l'apprenant, n'a jamais permis de comprendre comment des candidats apprenants (Bange 1992) s'y prennent pour s'approprier la compétence globale de membres d'une nouvelle communauté avec laquelle ils entrent en contact en participant à des interactions ordinaires. Une approche interactionniste, c'est-à-dire l'observation et l'analyse des échanges qui occupent les apprenants au sein de la communauté en question, permet de suivre non seulement les initiatives dans la mise en place des formes linguistiques, mais aussi, et en même temps, la constitution d'une compétence plus globale de type socio-culturel que certains appellent la socialisation en langue étrangère (Roberts 1999). C'est l'approche qui a été adoptée pour suivre pendant un an la vie d'une CLasse d'INitiation au français (CLIN) pour les enfants étrangers inscrits à l'école élémentaire dans le $20^{\text {ème }}$ arrondissement de Paris.

Après avoir exposé le cadre théorique dans lequel cette étude prend place, on présentera, à partir d'extraits de discours recueillis dans la classe, un aperçu des échanges langagiers qui cadrent et modèlent l'entrée dans la langue et la culture nouvelle. On essaiera, ce faisant, de montrer que, d'une part, acquérir une langue autre que sa langue maternelle ne saurait s'effectuer, et donc être analysé, dans le vide environnemental, et que, d'autre part, l'étude des interactions et discours construits en situation peut nous aider à appréhender comment s'élabore conjointement et en contexte cette compétence nouvelle, cette adaptation à la vie d'une nouvelle communauté dans ses aspects socio-culturels et linguistiques.

\section{Cadre théorique et choix méthodologiques}

\subsection{La parole échangée comme lieu d'élaboration linguistique et sociale}

Cette étude des activités interactionnelles se situe au carrefour de certains travaux anthropologiques (Schieffelin et Ochs 1986, Ochs et Schieffelin 1995), des 
études psychologiques sur le rôle de la médiation dans le développement langagier (Vygotski 1935/85, Wertsch 1985, Bruner 1983, Bruner 1991) ainsi que de l'interactionnisme. Ces conceptions de l'acquisition langagière ont en commun une vision holistique des activités humaines et, par conséquent, un décloisonnement des approches disciplinaires. Elles insistent sur le rôle de la construction collective des discours (Gumperz 1992), du contexte social (Duranti et Goodwin 1992) et des savoirs (Lave 1993) dans l'interaction. Elles ont ainsi suscité, en relation avec l'analyse de l'interaction verbale et, en particulier, de ce qui a été appelé la conversation, une réflexion spécifique sur la communication et l'apprentissage de la communication. Le discours y est conçu comme cadre de socialisation et d'« entrée dans la culture» (Bruner 1996). Et, en même temps, il apparait comme produit de la culture, elle-même élaborée et constamment façonnée par la communauté sociale.

Dans le cadre de l'appropriation langagière en général, l'influence des auteurs sus-nommés est notable dans le cadre des travaux sur le développement du langage chez l'enfant (François 1969, François, Hudelot \& Sabeau-Jouannet 1984, Hudelot 1993, Hudelot \& Vasseur 1997). En L2, les travaux se sont un moment focalisés sur les séquences de négociation de formes conçues comme manifestations de processus socio-cognitifs d'hétéro- et d'auto-régulation (Frawley et Lantolf 1985, Py 1990, Dausendschön-Gay \& Krafft 1991, Bange 1992, Vasseur 1995). Ils s'orientent actuellement vers un changement d'échelle et une diversification. On étudie des discours plus longs accompagnant et structurant des pratiques sociales plus diversifiées (Bremer et al. 1996, Arditty \& Vasseur 1999). Epousant cette approche, l'analyse des échanges en classe de langue 2 s'est intéressée au déroulement et aux enjeux de l'interaction dans la classe (Bange 1992, Barthomeuf 1991, Cicurel 1996, Gajo et Mondada 1998, Pekarek-Doehler 2002).

L'étude proposée ci-dessous de l'acquisition langagière en CLIN comme construction d'une compétence communicative complexe au sein de pratiques socioculturelles s'inscrit dans ce mouvement d'analyse qualitative déjà pratiquée pour d'autres situations. Elle se donne pour cadre ce que Ochs et Schieffelin (1995: 78) appellent l'écologie communicative. Ce terme désigne, pour un locuteur donné, les occasions de participation à des activités sociales et culturelles et la valorisation qui en découle pour l'individu et son discours. Rendre compte du travail d'appropriation socioculturelle et linguistique chez les élèves de CLIN consistera donc à s'interroger sur la participation et les discours des différents partenaires dans les différents contextes et activités de la classe.

\subsection{Données et procédure de recueil}

Les données qui ont été analysées font partie d'un ensemble d'enregistrements audio réalisés régulièrement une fois par semaine pendant une année scolaire dans une CLIN à Ménilmontant (Paris 20 ème). Ces enregistrements sont, dans une perspective ethnographique (reprenant la démarche de Gumperz 1982 par exemple), complétés par des notes prises au cours des enregistrements et concernant l'environnement, les circonstances et les autres systèmes sémiotiques de la communication dans la classe. Précisons que, dans l'esprit de l'approche ethnographique, on observe et on analyse ici la vie d'une classe donnée, dans une école donnée, avec un enseignant donné et qu'on 
ne la présente pas comme un modèle. Néanmoins, cette approche, au plus près des données particulières, se veut attentive aux procédures discursives récurrentes pour organiser le sens et l'apprentissage, attestées dans d'autres situations interculturelles et interlingues proches. En ce sens, elle permet de se poser, autrement que par une approche formelle et quantitative, la question: comment les enfants de CLIN apprennent-ils le français?

\subsection{Observables et outils d'analyse?}

On sait que dans la CLIN, et cela au moins trois à six heures par jour en moyenne, le français langue 2 est la langue de communication dominante. Deux aspects fondamentaux de la situation sont particulièrement déterminants : l'extrême hétérogénéité des âges, niveaux, origines et environnements de vie des enfants et la multiplicité des objets, activités et domaines qui doivent faire l'objet d'un apprentissage (Chiss 1999). La langue elle-même est objet d'apprentissage et en même temps outil, non seulement pour l'apprentissage de la langue, comme dans la classe de langue étrangère, mais aussi pour les autres apprentissages. Dans les pratiques de ces élèves qui découvrent à la fois une autre culture et une autre culture scolaire en même temps qu'une autre langue, on observe donc, encore plus que chez les élèves français, un véritable emboîtement des apprentissages dont l'interaction entre les différents protagonistes est le moteur et la socialisation nouvelle l'enjeu crucial.

Les stratégies des jeunes enfants étrangers au groupe sont d'abord, comme l'a montré Wong-Fillmore (1976, 1989), d'ordre social et socio-communicatif. Dans la CLIN, où les enfants sont âgés de 7 à 12 ans, l'appartenance au groupe est d'abord imposée par l'inscription scolaire. Mais cette appartenance reste à construire et l'enjeu est considérable. On peut même postuler que cette construction plus ou moins rapide et efficace conditionne les apprentissages. Les discours de la classe en sont le lieu et l'outil. Par la parole partagée au fil des jours, les membres de la communauté-classe négocient et construisent leur compréhension d'un ordre social qu'ils s'approprient peu à peu. On voit, au fil du temps, se constituer une communauté de membres (Garfinkel et Sacks 1970, Coulon 1987, 1993) autour d'une histoire collective d'apprentissage. Dans ce contexte complexe, l'apprentissage de la langue nouvelle, même s'il fait l'objet de séquences pédagogiques focalisées et de séquences de reformulations contrôlées, n'est jamais longtemps isolé de l'expérience commune de la parole partagée. Pour essayer de comprendre comment se construisent les compétences à communiquer dans et par l'interaction en groupe, on ne peut donc se limiter à observer les formes linguistiques (unités ou structures) considérées isolément $\mathrm{du}$ contexte linguistique et interactionnel. On doit s'intéresser aux aspects collaboratifs du discours collectif qui se développe dans la communauté. Plus particulièrement, on interrogera ici différents types de séquences interactionnelles pour se demander comment la parole des partenaires, dans ses formes discursives et linguistiques, attendues ou non, reprises ou non, modifiées ou non, contextualisées ou non, à la fois configure et est configurée par les activités diverses de la classe dans lesquelles elle s'insère de façon fonctionnelle. Nous considérerons ces liens entre initiatives et genres discursifs dans quatre types de séquences: des séquences de 
constitution du groupe centrées sur l'intersubjectivité, des séquences de mise en place des routines scolaires, des séquences d'élaboration de savoirs scientifiques et techniques et des séquences de travail sur la langue.

2. Analyse : le discours collectif de la classe comme lieu d'élaboration d'une identité sociale, de savoir-faire scolaires et de savoirs et comme cadre de la construction des compétences linguistiques

\subsection{La mise en place d'une communauté de membres et d'une intersubjectivité qui se construit discursivement}

Cette construction passe d'abord par un partage des expériences de chacun. Les différentes activités ou événements de la classe sont souvent l'occasion, pour les élèves ou pour l'enseignante $(\mathrm{MC})$, de faire référence à leur vie passée, à leur environnement familial actuel, à des événements vécus par eux hors de l'école. Souvent, dans un échange spontané comme dans l'extrait ci-dessous d'une classe de premier trimestre, les élèves prennent la parole, par exemple en se référant à la vie familiale ou culturelle de l'autre, en posant des questions à l'autre - y compris l'enseignante — ou sur l'autre. Ci-dessous, les pleurs de T, une élève sri-lankaise, suscitent chez l'enseignante une explication sur la situation personnelle de T, ce qui déclenche des demandes de clarification de la part d'un autre élève. Cette initiative inattendue permet à la fois de faire partager au groupe le vécu familial d'un de ses membres et pour cela d'activer des ressources linguistiques mal maitrisées comme la désignation des liens de parenté : c'est quoi la tante?, T elle a pas de maman?, ou les marques d'identité comme le nom : et toi t'habites à la France? pasque le nom de $M C$ (nom de $M C)$ :

(T. qui s'est fait bousculer à la récréation rentre en pleurant, MC l'enseignante prend la parole $)^{1}$

1.MC : T a mal au bras droit + elle a eu un accident + sa tante m'a raconté $(\ldots)$

2.G : c'est quoi la tante?

3.MC : c'est la sœur de papa + on l'a fait dans l'arbre (généalogique) Gunai tu as

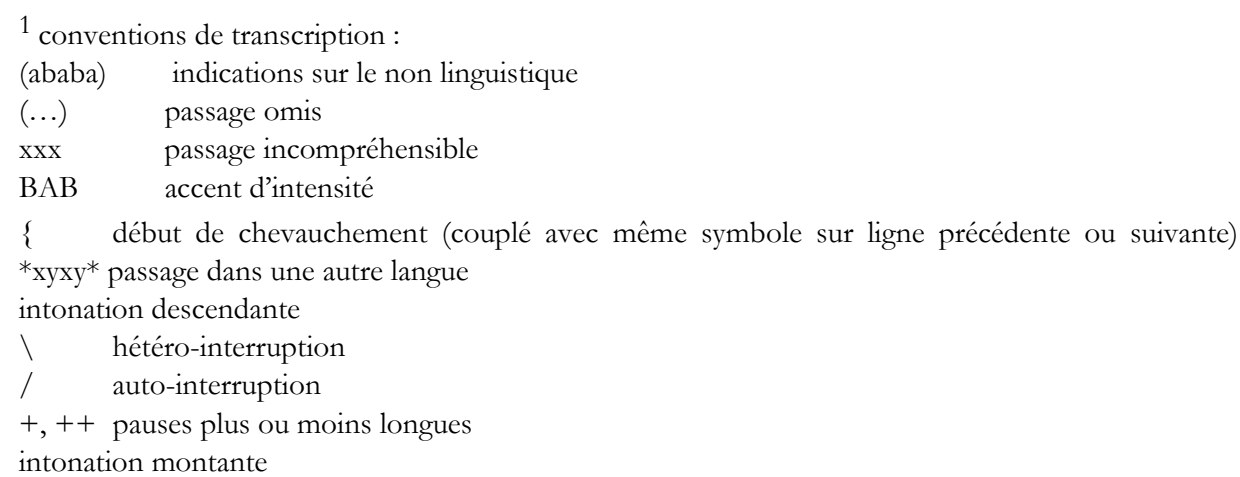

Les locuteurs sont identifiés en début de ligne par l'initiale de leur nom, El ou Els renvoie à un ou plusieurs élèves. 
En CLIN, l'apprentissage du français passe par la socialisation en français

oublié

4.F : T elle a pas de maman?

5.MC : si mais elle n'est pas là . T habite avec sa tante

6.(......)

7.F (à MC) : et toi t'habites à la France ? + pasque le nom de MC (nom polonais)

8.MC : ah oui j'habite en France pasque mon grand'père est venu d'un autre pays la

Pologne (elle montre sur la carte et leur dit bonjour en Polonais) M (yougoslave) il

peut comprendre y a des mots qui sont presque pareils

9.D : moi c'est Chine + bonjour comme ça [niho] (....)

De même, et surtout, les discours échangés permettent de se constituer une mémoire collective, à travers par exemple les restitutions et récits des activités menées collectivement et rappelées, ou bien prévues et annoncées par l'enseignante. Ces discours collectifs sont alors majoritairement structurés par les questions, les reprises et les explications de l'enseignante chez qui l'usage du nous/on apparait comme la marque et en même temps l'outil constructeur de la conscience et de la catégorie du groupe-classe. Ces discours en nous peuvent alors, comme on peut le voir dans l'extrait suivant, être confrontés au discours en je d'un élève, G, qui propose sa propre expérience en contraste :

1.MC : quand nous sommes sortis du métro + qu'est-ce qu'on a fait?

2.Els : xxx le Pont, le pont-neuf

3.MC : nous avons traversé le Pont-Neuf

4.Els : et les photos MC

5.MC : et on a fait des photos, ya des gens des touristes ils ont fait des photos avec vous $+(\ldots)$ et on est descendu ++ dans la rue non + est-ce qu'en descendant on a marché sur la Seine, non on a marché sur le ? sur le?

6.R : quai

7.MC : bien $\mathrm{R}+$ bon qu'est-ce qu'on a vu?

8.G : $x x x$ pour les pompiers comment s'appelle?

9.Els : xxx restaurant

10.MC : xxxx une péniche-restaurant

11.G : moi j’ai regardé dans cuisine

12MC : oui on a vu les cuisines (...)

C'est l'occasion aussi, pour l'élève, de s'approprier l'espace commun quotidien, en le nommant, en le découpant, en le dessinant et en signalant même, comme G cidessous, les problèmes que peuvent poser les objets intermédiaires de communication (plans, dessins, métaphores) utilisés par l'enseignante: (10) moi je sais pas que c'est l'escargot)

1.MC : combien de ponts y a-t-il sur la Seine?

2.Els répondent dans le brouhaha

3.MC : $\mathrm{xxxx}+$ et combien d'arrondissements dans Paris ?

4.Els : vingt 
Marie-Thérèse Vasseur

5.MC : et nous on habite dans le combien ?

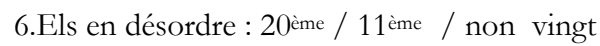

7.MC : et ça ressemble à quoi l'ordre des arrondissements de Paris sur le dessin?

8.G : l'esca(r)got

9.MC : oui l'escargot (elle dessine un escargot)

10.G : moi je sais pas que c'est l'escargot

11.MC : et nous on habite ici + qui est le vingtième (montre son dessin de Paris et ses arrondissements)

12.H : MC + A. il dit onzième

13.C : oui regardez (dessine le plan du quartier autour de l'école) l'école est ici + je vais traverser la rue (montre le carrefour entre plusieurs boulevards) ici c'est quoi ?

De même, l'évocation des activités à prévoir est pour les enfants l'occasion d'élaborer ou de réactiver un agenda qu'ils doivent s'approprier, et, en même temps, une source de réactions individuelles quant à ces activités. Ainsi, dans l'extrait suivant, deux élèves construisent leurs réactions verbales autour du contraste nous-on/je, comme en écho pour $\mathrm{H}$ : oui j'ai la photo d'Algérie de la classe, et pour soit s'inclure soit s'opposer pour G : c'est nous on fait gâteaux? moi non (= je ne fais pas le concert) + $\operatorname{regard}[e])$.

(Broubaha généralisé à la fin d'une activité)

1.MC : bon comme ça fait un petit moment qu'on n'a pas chanté je voudrais que nous répétions un peu la chanson de Claire + pasque cet après-midi on va rester un peu dans la cour pour la photo de classe

2.H : oui j’ai la photo d'Algérie de la classe

3.G : MC c'est nous on fait gâteaux ? (participation à un petit commerce de gatteaux pour financer la fête de l'école)

4.MC : non demain nous ne vendons pas des gâteaux pasque nous avons xxxx les CM2 + demain après le sport les CM1 et CM2 vont nous faire un concert de percussions pour Musicora

5.G : MC moi non + regard[e] (= je regarde)

6.MC : oui

7.G : moi non + ouf $(\ldots$.

Les initiatives - inattendues - de l'élève, dans ce que l'on présente souvent comme une mécanique fort contraignante du discours de la classe (type IRE: Initiative / Réaction / Evaluation, cf. Sinclair et Coulthard 1974), peuvent prendre la forme directe de questions-demandes d'information ( $F: T$ elle a pas de maman ?), de questions sur la langue ( $\mathrm{G}$ : c'est quoi la tante?), d'assertions-rapports d'expérience ( $\mathrm{G}$ : moi j'ai regardé dans cuisine, $\mathrm{H}$ : oui j'ai la photo d'Algérie de la classe), de demandes de confirmations (c'est nous on fait gâteaux?), d'expression de goûts et choix personnels $(\mathrm{G}: M C$ moi non + regard $[e]$ (= je regarde)) mais aussi de sollicitations indirectes (moi je sais pas que c'est l'escargot) qui influent plus ou moins sur le cours du discours collectif de la classe, puisqu'elles suscitent réponses et réactions de l'enseignante et parfois des autres élèves. L'élève qui prend l'initiative discursive et thématique prend aussi, on le 
constate, l'initiative d'utiliser les outils linguistiques dont il dispose, ce qui peut avoir pour effet d'en confirmer ou d'en infirmer l'usage en fonction des réactions de l'enseignante, comme on le voit dans ce qui suit.

\subsection{Les discours collectifs, lieu de construction des rituels et savoir- faire scolaires}

Une part importante de l'expérience collective se structure autour du discours sur les pratiques scolaires que l'enseignante cadre par des rappels réguliers. Cela recouvre pour l'élève des routines telles qu'inscrire la date au tableau, se repérer dans l'emploi du temps et les différentes activités, demander un nouveau cahier, coller la feuille des activités sur le cahier personnel etc. Ce cadrage n'interdit pas alors, dans le discours conjoint, l'expression de soi, l'humour et la connivence. Il admet aussi de la part de l'enseignante des interventions parenthétiques sur la langue, que les enfants reprennent et ajustent au contexte discursif :

(Exercice écrit de questions/ réponses à partir du dialogue de la leçon)

1.MC : prenez vos cahiers $+\mathrm{K}$ tu mets la date au tableau

2.K s'exécute

3.R à MC : MC cahier fini

4.MC : mon cahier?

5.R : fini

6.L : est fini

7.MC (à R): R j'écoute + est ?

8.R : fini

9.MC : non EST fini

10.R : est fini

11.MC : est fini + je vais t'en donner un autre + (à tous) on sort son cahier on écrit la date $\backslash$

12.El : on colle

13.MC : on colle le petit texte

14.El : le cahier (brouhaha)

15.MC : jeudi 29 mars +

16.MC (à $\mathrm{R}$ pour noter les cahiers neufs qu'elle distribue) : c'est le deuxième? 17.R : oui

18.H : MC on a droit de colorier les cousins de Ratus?

19.F : XXX

20.K : ah la la beaucoup de colle

21.F : la colle è(lle) colle pas

22.G : ah aujourd'hui y'en a de la musique c'est bien + y a la musique la

23.MC : euh c'est pas y'en a de la musique c'est IL Y A

24.L : il y a (brouhaha)

25.H : MC moi mon cahier ya deux

26.El : MC moi comme H 
Marie-Thérèse Vasseur

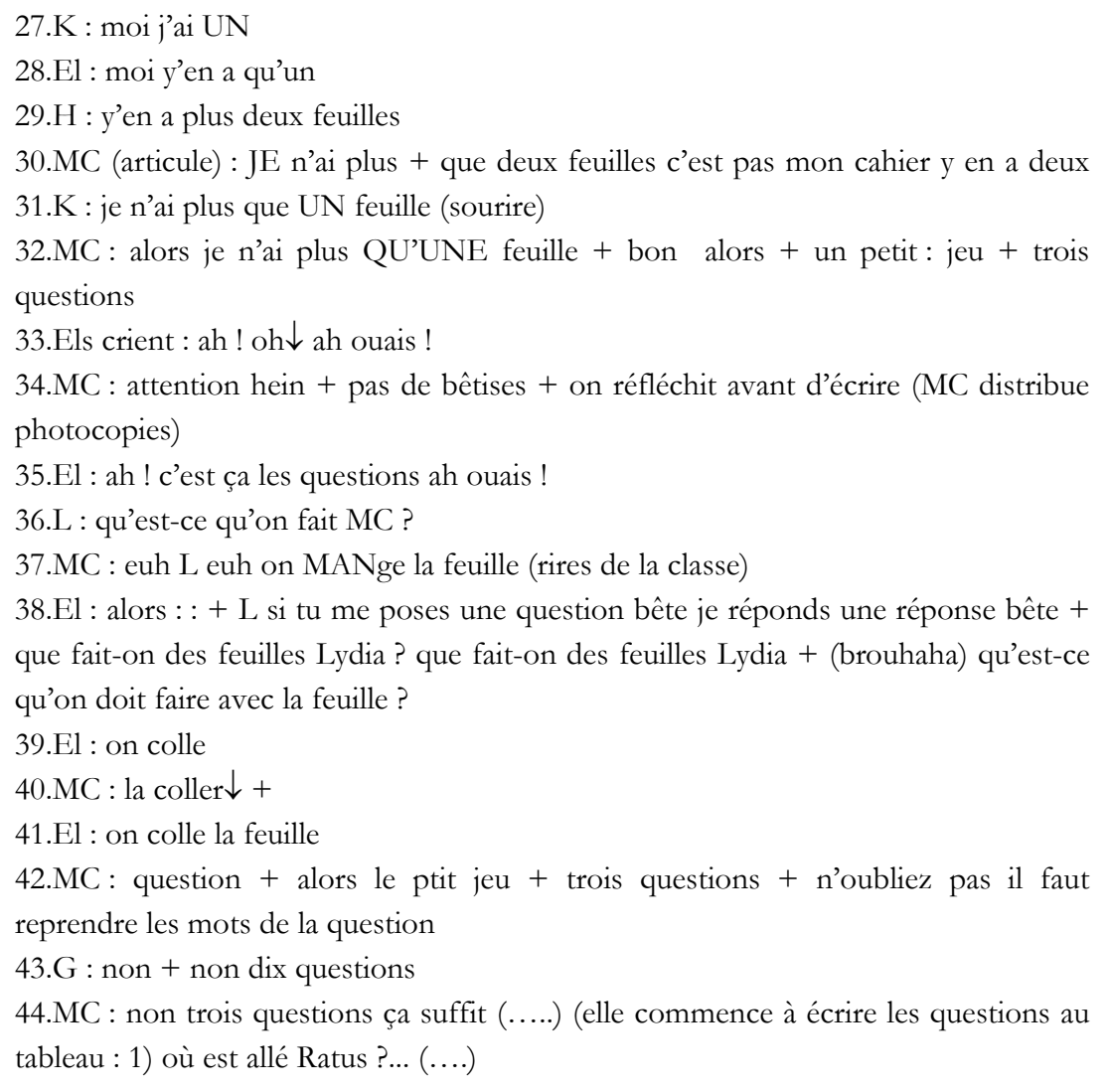

Dans cette activité très cadrée qui consiste, après une activité orale sur de petits dialogues, à passer à l'écrit sur les cahiers, l'élève prend souvent la parole et montre qu'il contrôle l'activité : il peut constater et indiquer ses problèmes avec les outils (cabier fini, la colle e(lle) colle pas, moi mon cabier y a deux), prendre les devants sur l'action à accomplir en la nommant (on colle), vérifier l'activité à accomplir et ses limites (qu'est-ce qu'on fait $M C$ ?, on a droit de colorier ?). Et constamment, en parlant des outils et des tâches, il manipule, teste, reprend les formes linguistiques qui lui permettent d'en parler.

Les initiatives des élèves sont aussi pour l'enseignante l'occasion d'introduire d'autorité, en tant qu'enseignante mais aussi expert en L2, des séquences de travail correctif sur la langue (usage de la négation : c'est $P A S \ldots$.., et de l'injonctif : répète) lors desquelles elle exige la reprise de sa reformulation. Ces séquences de travail se développent à différents niveaux d'implication et d'appropriation selon les enfants. La correction est soit reprise sans plus par l'élève concerné, assisté par un autre (3-11), soit répétée en écho par un autre élève (22-24), soit encore reprise et contextualisée par K, l'un des élèves concernés (27-31).

Ce mode et ce schéma d'hétéro-correction, qui peut être perçu comme une rupture agressive de l'échange, voire de la tâche en cours, est l'occasion saisie par certains élèves, qui s'y soumettent, de se réapproprier immédiatement et à leur propre 
usage le modèle syntaxique imposé. C'est ce que fait $\mathrm{K}$, qui à la fois s'approprie la structure syntaxique corrigée et ajuste au contexte concret le contenu de son énoncé. Cette réaction nous montre bien que le travail sur la langue, qui est une décontextualisation (par l'enseignante), peut être l'objet d'une bifocalisation (forme et adéquation du contenu) de la part de l'élève. Dans cette séquence, le contexte, qui peut se formuler ainsi: 'je n'ai plus qu'une page dans mon cahier, il m'en faudra bientôt un nouveau', construit l'énoncé tout en étant en même temps construitreconstruit verbalement par les discours mêmes des interlocuteurs (Duranti et Goodwin 1992).

\subsection{Les discours collectifs, voies d'échange, de confrontation et de structuration des savoirs}

Dans l'extrait suivant, tiré d'une leçon sur les phénomènes atmosphériques, il s'agit d'abord pour l'enseignante, en s'appuyant sur le questionnement, de faire expliciter et partager des expériences atmosphériques vécues par tous: le vent, la pluie, l'orage, le tonnerre, la foudre. L'objectif de cette activité est double : rendre les élèves capables de parler de ces phénomènes en leur fournissant les termes qui désignent ces phénomènes (neige, tonnerre, éclair), mais aussi, en même temps, aider les enfants à passer du discours réactif et subjectif qui est le leur à un discours plus objectivisant et scientifique. Ce partage institutionnel des rôles pourrait se manifester de façon assez schématique par une dissymétrie systématique des échanges. Or, on observe que la recherche collective des mots pour dire ce vécu, évoquer son ressenti et comprendre la réalité donne au discours un tour nettement collaboratif et que cet objectif global de compréhension-explication, mettant le travail linguistique au service de l'action entreprise de construction de savoirs, fait passer la focalisation linguistique au second plan.

$1 \mathrm{MC}$ : qu'est-ce que c'est la neige ? + quand est-ce qu'il neige ? quand il fait chaud ou froid?

2Els : froid

$3 \mathrm{MC}$ : et quand il fait froid quand il pleut qu'est-ce qu'il tombe?

4Els : de l'eau

$5 \mathrm{MC}$ : et quand il fait froid les petites gouttes de pluie se changent en neige + ya des ptites gouttes de neige (elle dessine au tableau des cristaux) et c'est des cristaux $6 \mathrm{R}$ : en Roumanie encore

$7 \mathrm{MC}$ : y a encore de la neige en Roumanie ? + donc la pluie et la neige c'est de l'eau + et hier on a entendu quelque chose dans le ciel de Paris + ça s'appelle comment quand on entend du bruit dans le ciel ?

$8 \mathrm{~K}$ : (dit un mot en arabe)

9MC (rires) : en arabe mais en français ? (Els cherchent, s'agitent) ++ le tonnerre $10 \mathrm{~K}$ : oui ça fait peur + MC y a deux choses MC ! ça vient rouge comme ça comme [ع] pleut

$11 \mathrm{MC}$ : oui alors il vient quelque chose

$12 \mathrm{~K}+\mathrm{F}$ : jaune $\mathrm{x}$ jaune 
Marie-Thérèse Vasseur

$13 \mathrm{MC}$ : alors qu'est-ce que c'est jaune dans le ciel ?

14A : ah oui jaune comme ça comme d'électricité

15MC : et on dit il ne faut pas être sous un arbre + ça s'appelle des éclairs (écrit au tableau) et c'est jaune en effet

$16 \mathrm{~K}$ : oui $\mathrm{MC}+$ fait comme ça (geste de gauche à droite et vers le bas) et ça fait peur

17R : MC comme Z

$18 \mathrm{~F}: \mathrm{y}$ a rouge vert rose et des verts des couleurs comme ça

19MC : A. on dit c'est dangereux il peut y avoir de l'électricité + une boule de feu qui tombe sur les maisons + alors on met des pointes sur les immeubles + par exemple on voit (montre par la fenêtre) les pointes pour protéger xxxxx ça s’appelle la foudre $\operatorname{xxxxx}+$ alors i peut y avoir aussi (dessine au tableau un arc-en-ciel) $20 \mathrm{~K}$ : oui comme ça MC (geste pour dessiner un éclair)

21A : MC en Algérie faut pas faire ça faut pas faire ça (insistant) (....)

Dans ce groupe que constitue la CLIN, une expérience comme celle de la foudre est partagée mais, étant donné les origines diverses des enfants, elle l'est à des degrés divers et sous des formes diverses. Il y a là dans le discours une ouverture possible et pour les élèves des occasions de prise de parole individuelle. Ils répondent aux questions en fournissant la réponse ponctuelle attendue : qu'est-ce qu'il tombe? / de l'eau. Ils contextualisent le discours de l'enseignante en proposant une localisation qui les intéresse : en Roumanie encore. Ils réagissent au phénomène évoqué (le tonnerre) en verbalisant leur réaction subjective : ça fait peur. Ce faisant, ils fournissent des indices de leur compréhension et de leurs savoirs. On remarque aussi que malgré leurs ressources limitées en français, les élèves collaborent activement pour convoquer à la fois leurs savoirs (MC y a deux choses, $M C$ ça vient rouge/ jaune xxx jaune, jaune comme ça comme d'électricité), leurs émotions (oui ça fait peur) et aussi les croyances qui leur sont liées (en Algérie faut pas faire ça).

Ces indications permettent à l'enseignante d'orienter le dialogue vers les éléments linguistiques non connus qu'elle propose à leur appropriation (le tonnerre, ça s'appelle les éclairs, ça s'appelle la foudre...), et vers des perceptions moins subjectives et plus 'scientifiques' de ces phénomènes. Les reformulations qu'elle produit alors ont deux fonctions : elles valident les interventions des élèves et en même temps peuvent en proposer, sans l'imposer, une autre mise en forme linguistique : y a encore dla neige en Roumanie?, on dit c'est dangereux il peut y avoir de l'électricité.

Ce qui frappe donc ici, c'est la convergence et la continuité des discours entre les participants.

L'enseignante s'attache à arrimer son discours questionnant et constructeur de concepts scientifiques et techniques (la foudre phénomène naturel et le paratonnerre protecteur contre le danger et donc la peur) au discours réactif limité mais chargé d'expérience et d'intérêt produit par les enfants. A travers ses questions, elle oriente et fait progresser le discours collectif. Mais loin de se concentrer sur la seule présentation d'unités lexicales (ça s'appelle des éclairs), elle s'efforce non seulement de laisser se développer les interventions, initiatives, recherches des élèves $(M C y$ a deux choses $M C$ ça vient rouge, jaune comme ça comme d'électricité...) mais aussi de faire avancer le thème à partir des éléments proposés par les élèves. Cette dynamique de la séquence tient au 
travail constant de reprise-reformulation des élèves et de l'enseignante, autour de termes qui passent des uns aux autres : vient, jaune ou électricité par exemple.

Ce travail qui s'appuie sur la continuité garantit la cohésion du discours collectif, la poursuite de l'objectif de compréhension-apprentissage de ces phénomènes et, indissociablement, il permet la présentation aux enfants de modèles linguistiques plus complexes et structurés. Il ne s'agit pas ici de recourir à un langage simplifié ou d'isoler pour les corriger des énoncés non conformes mais, en reconnaissant les élèves comme des interlocuteurs légitimes, de leur accorder une place égale de participant à l'interaction et ainsi d'établir pour eux, peu à peu et de façon intégrée par cette approche même, le contact avec des savoirs et des discours complexes.

\subsection{L'apprentissage de la langue : imposition, appropriation et jeu des places}

A certains moments, les discours de la classe se focalisent aussi sur la langue. Ces séquences spécifiques, ou leçons de français oral et écrit, se caractérisent par une forte dissymétrie dialogique, mais certains élèves s'autorisent néanmoins à sortir d'un rôle et d'un schéma discursif figé et contraignant, comme on le constate dans l'extrait suivant :

(leçon sur les pronoms personnels)

1. MC : est-ce que vous avez un goûter à 10 heures ?

2.G : moi y a pas de goûter

3.MC : JE N'AI PAS de goûter (elle écrit le modèle au tableau) + MOI je veux pas de ce mot-là $(. . .$.

4.MC (à $\mathrm{A}$ qui ne sait pas répondre à sa question) : oui euh alors je vais reprendre ça pasque + écoute bien + j'ai demandé à $G+[\varepsilon$ səkə] tu as un goûter à dix heures ? G m'a répondu + non + JE n'ai pas de goûter + par contre j'ai demandé à Sacha + Sacha est-ce que tu as un goûter à dix heures (écrit au tableau)

5.Els dont $\mathrm{S}$ : oui j'ai un goûter/ oui je suis un [gote]

6.MC (en écrivant) : oui

7.Els dont S : oui j'ai / MOI je un goûter

8.MC : J'AI + ouvre ta bouche pasqu'entre je j'ai on entend pas très bien + OUI + J'AI (écrit en même temps au tableau)

9.G : MC (il pointe le j’) + [3ə] ?

10.MC : $[3 \varepsilon]+\mathrm{G}$ attention regarde bien (elle repasse l'apostrophe à la craie) + J'AI

+ un goûter

11.Els lisent : j’ai a goûter/un goûter

12.W à sa voisine : c'est pas [a] + UN hii ! (rires)

13.MC: $(. . .$.$) alors maintenant \mathrm{A}$ je te demande $+[$ Esəkə] + tu as un crayon ?

14.A : oui $+\mathrm{je} / \mathrm{ji}$

15.MC : j’ai

16.A : moi

17.MC : non! je veux pas de moi + poubelle le moi/ à la poubelle moi 


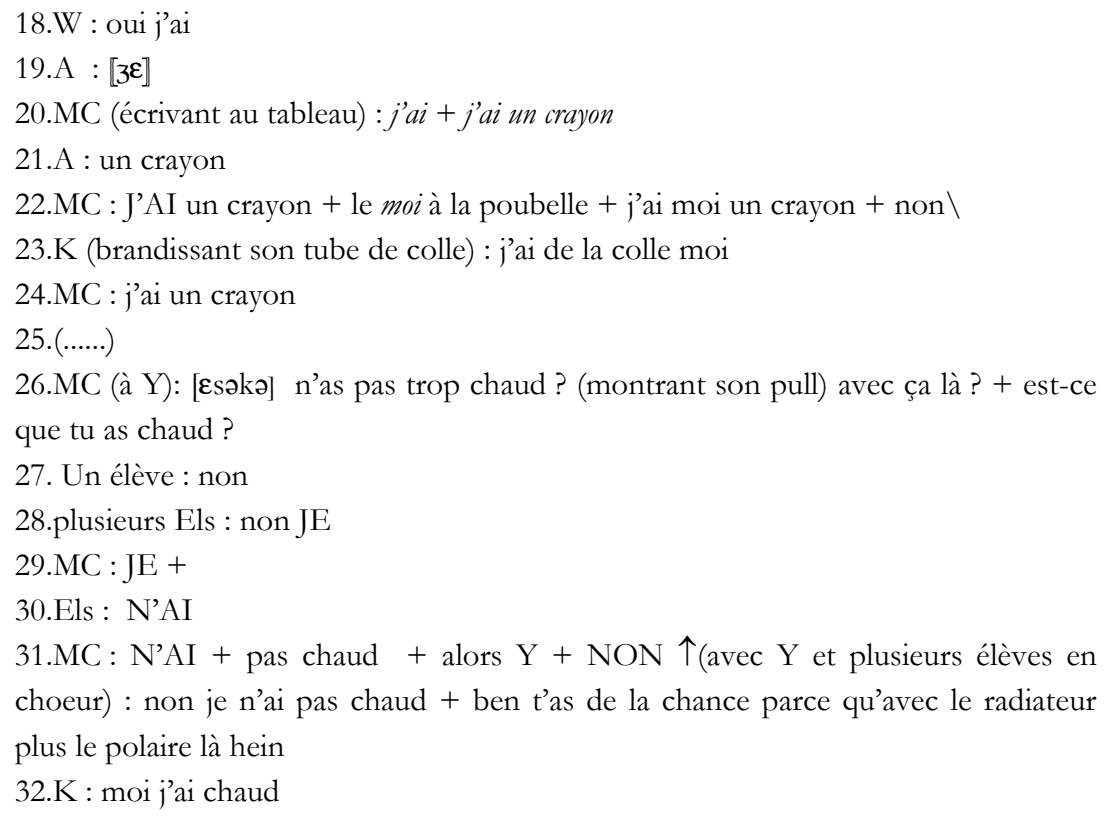

Différents mouvements discursifs se dessinent donc dans les discours des élèves. Soit ceux-ci acceptent d'entrer dans le genre didactico-métalinguistique du discours de l'enseignant : c'est le cas quand $G$ se glisse activement dans le rôle imposé de l'élève en répondant aux questions posées et en prenant même l'initiative (9-10) de poser une question pointue sur l'usage ou la prononciation de j' (pour je). Soit ces mêmes élèves divergent en quittant le niveau 'méta' de l'échange pour réinvestir personnellement la structure travaillée dans un usage immédiatement contextualisé. C'est le cas de K qui, au terme d'un échange didactique très encadré, prend la liberté de s'exprimer sans avoir été sollicité. Son énoncé (23) reprend avec souplesse le modèle morpho-syntaxique travaillé et l'ancre dans la réalité. Lorsqu'il s'écrie : j'ai de la colle moi, il montre son tube de colle, et lorsqu'après un échange avec un autre élève il ajoute moi j'ai chaud (32), il s'inscrit toujours dans sa réalité et par contraste avec l'élève interrogée. Une autre forme enfin de participation marquée de l'élève se manifeste ici. C'est celle du changement de place: W s'adresse sur le ton de la plaisanterie à une autre élève pour la corriger : c'est pas [a] $+U N$ (rires) (12). Par cette initiative de prise de parole et la place discursive inattendue qu'elle s'attribue dans l'échange, elle montre qu'elle aussi peut se focaliser sur les formes linguistiques et les contrôler.

Ces modes variés d'intervention de l'élève indiquent que ce dernier peut choisir entre différentes places : celle attendue d'élève qui répond et répète quand on le lui demande, celle d'apprenant qui, en initiant des questions, montre qu'il organise et contrôle son apprentissage, celle d'expert-enseignant, quand il contrôle les productions des autres élèves, en même temps que celle, double, de communicateurapprenant qui veille à maintenir un lien entre le travail sur la langue et l'ancrage contextuel de ses discours. Il exploite ainsi efficacement les ressources de l'interaction pour s'entraîner dans la langue. Ce faisant, il adapte ses savoir-faire linguistiques à la situation, c'est-à-dire qu'il apprend. 


\section{Conclusion}

La perspective selon laquelle on vient d'aborder les discours dans la CLIN fait apparaitre l'apprentissage de la langue comme s'insérant, aux différents niveaux des différents apprentissages, au sein de l'ensemble des activités discursives à l'école. Trois niveaux principaux de circulation et d'usage des discours ont été distingués ici : les discours constitutifs de la communauté où l'enseignant et les élèves évoquent et restituent des expériences particulières et collectives, les discours lors desquels les élèves s'approprient les rituels de la classe et apprennent en quelque sorte leur 'métier d'élève' en mettant en place des moments et formules plutôt stéréotypées d'actions et de discours, les discours constructeurs de savoirs où la continuité est forte entre les discours des participants qui collaborent, et enfin les discours sur et pour la langue où la focalisation est le fondement de l'activité mais où les élèves peuvent se positionner différemment du point de vue de leur participation. A ces différents niveaux néanmoins l'apprentissage de la langue est présent sous des modalités différentes. Les contextes discursifs qui peuvent ouvrir sur de l'apprentissage sont en effet variés : le genre (leçon de langue, leçon de choses, tâche pratique...), le thème plus ou moins évocateur comme l'orage, les rôles et places des locuteurs et leurs initiatives de parole, l'information pratique à communiquer...

Les enfants de la CLIN observée apprennent ainsi la langue non pour ellemême et en elle-même, mais en participant à divers types de tâches discursives dont les fonctions consistent à apprendre à devenir membre d'une communauté : le groupe classe, à s'initier aux savoir-faire scolaires, à apprendre à élaborer des savoirs en reconstruisant son expérience, à apprendre à jouer le rôle attendu de l'élève mais aussi à le dépasser pour occuper une place d'interlocuteur reconnu dans le discours collectif y compris lorsqu'il a pour objet la langue 2 à apprendre.

On a pu voir ici à travers quelques extraits du discours collectif d'une classe donnée que, malgré la présence massive du discours de l'enseignante, certains enfants (pas tous, pour différentes raisons) prennent une part active dans cette construction complexe. C'est même parce que l'enseignante les considère comme interlocuteurs à part entière qu'ils peuvent optimiser leurs places discursives, en adaptant à la situation qu'ils contribuent à construire les contenus et les formes de leurs discours, qu'ils entrent donc dans une culture nouvelle et apprennent la langue 2.

vasseurm@vjf.cnrs.fr 


\section{BIBLIOGRAPHIE}

ARDITTY, J. (1987) : «Une leçon de conversation», dans J. ARDITTY (dir.), Paroles en construction, Encrages 18-19, Université Paris VIII, Vincennes à Saint Denis, 45-68.

ARDITTY, J. \& M.-T., VASSEUR (1999) : «Présentation », Langages 134, 3-19.

BANGE, P. (1992) : «A propos de la communication et de l'apprentissage en L2, notamment dans ses formes institutionnelles », AILE 1, 53-86.

BARTHOMEUF, J. (1991) : "Asymétrie et apprentissage dans les activités de groupe en classe » dans C. RUSSIER, H. STOFFEL \& D. VERONIQUE (dirs.) Interactions en langue étrangère. Aix-en-Provence, Publications de l'Université de Provence, 249-258.

BREMER, K., ROBERTS, C., VASSEUR, M.-T. et al (1996) : Achieving intercomprehension : discourse in intercultural encounters. London, Longman.

BRUNER, J. S. (1983) : Le développement de l'enfant : Savoir faire, savoir dire. Paris, Presses Universitaires de France, coll. Psychologie d'aujourd'hui.

BRUNER J. S. (1991) : ...car la culture donne forme à l'esprit, de la révolution cognitive à la psychologie culturelle. Paris, Eshel.

BRUNER, J. (1996) : L'éducation, entrée dans la culture. Paris, Retz.

CHISS, J.-L. (1999) : «Eléments de problématisation pour l'enseignement-apprentissage du français », dans CHISS J. L. \& D. BOYZON-FRADET, Enseigner le français en classes hétérogènes. Paris, Nathan.

CICUREL, F. (1996): "Hétérogénéité des dires dans une situation d'enseignementapprentissage » dans SOUCHON M. (dir.). Pratiques discursives et acquisition des langues étrangères. Besançon, Université de Franche-Comté.

COULON, A. (1987) : L'ethnométhodologie. Paris, Presses Universitaires de France, coll. Que saisje?

COULON, A. (1993) : Ethnométhodologie et éducation. Paris, Presses Universitaires de France.

DAUSENDSCHÖN-GAY, U. \& U., KRAFFT (1991) : «Rôles et faces conversationnels : à propos de la figuration en 'situations de contact », dans RUSSIER G., H. STOFFEL \& D. VERONIQUE (dirs.): Interactions en langue étrangère, Publications de l'Université de Provence,

DURANTI, A. \& C., GOODWIN (éds) (1992): Rethinking Context : language as an interactive phenomenon. Cambridge : Cambridge University Press.

FRANÇOIS, F. (1969) : «Ebauches d'une dialogique», Connexions 38. Langage en situation. Pratiques sociales et interaction. EPI éditions, 61-87.

FRANÇOIS, F., C., HUDELOT, E., SABEAU-JOUANNET (1984) : Conduites linguistiques cher. le jeune enfant. Paris, Presses Universitaires de France.

FRAWLEY, W. \& LANTOLF, J. (1985): «Second Language Discourse: A Vygotskyan Perspective ", Applied Linguistics 6-1. 
GAJO, L. \& MONDADA, L. (1998) : «Contexte, activité discursive et processus d'acquisition, quels rapports? » dans SOUCHON M (dir.), Pratiques discursives et acquisition d'une langue étrangère. Besançon, Université de Franche-Comté.

GARFINKEL, H.\& H., SACKS. (1970) : «On formal structures of practical actions », in D. J. MACKINNEY \& E. A TIRYAKIAN (eds.), Theoretical Sociology, Appleton-Century Crofts, New-York, 337-366.

GUMPERZ, J. (1982) : Discourse strategies, Studies in interactional sociolinguistics. London, Longman.

GUMPERZ, J. (1992) : «Contextualisation \& understanding», in DURANTI A. \& GOODWIN C. (eds.): Rethinking Context : language as an interactive phenomenon, Cambridge, CUP, 230-252.

HUDELOT, C. (1993) : «Du noviciat de l'expert. Etayage, débrayage et cafouillages dans un dialogue adulte-enfant », Cabiers de Linguistique Sociale 23, 51-83.

HUDELOT, C. \& M.-T., VASSEUR (1997) : «Peut-on se passer de la notion d'étayage pour rendre compte de l'éléboration langagière en L1 et en L2 ? ", Cabiers d'Acquisition et de Pathologie du Langage 15, 115-141.

LAVE, J. (1993) : «Situating learning in communities of practice», in L. B. RESNICK, J. M. LEVINE \& S. D. TEASLEY (eds.) : Perspectives on Socially Shared Cognition. American Psychological Association, Washington D.C.

OCHS, E. et B., SCHIEFFELIN (1995): "The Impact of Language Socialisation on Grammatical Development », in FLETCHER \& MCWHINNEY, The Handbook of Child Language, Oxford, Blackwell Publisher, p. 73-95.

PEKAREK DOEHLER, S. (2002) : «Formes d'interaction et complexité des tâches discursives : les activités conversationnelles en classe de L2», dans CICUREL F. \& D. VERONIQUE (dirs.) : Discours, action et appropriation des langues. Paris, Presses de la Sorbonne Nouvelle.

PY, B. (1990) : «Les stratégies d'acquisition en situation d'interaction », dans GAONAC'H D. (dir.) : Acquisition et utilisation d'une langue étrangère. L'approche cognitive. Le Français dans le monde, Recherches et applications, 81-88.

ROBERTS, C. (1999) : «Acquisition des langues ou socialisation dans et par le discours ? Pour une redéfinition du domaine de recherche sur l'acquisition des langues étrangères ». Langages 134, 101-116.

SCHIEFFELIN, B. \& E., OCHS (eds.) (1986) : Language Socialization across Cultures. New-York, Cambridge University Press.

SINCLAIR, J. \& M., COULTHARD (1974): Discourse analysis. Cambridge, Cambridge University Press.

VASSEUR, M.-T. (1993) : «Gestion de l'interaction, activités métalangagières et apprentissage en langue étrangère », dans U., DAUSENDSCHÖN-GAY \& U., KRAFFT (dirs.), Acquisition Interaction en Langue Etrangère 2, 25-59. 
Marie-Thérèse Vasseur

VASSEUR, M.-T. (1995) : «Le rôle de l'interlocuteur natif dans l'interaction exolingue », Cabiers de Praxématique 25, 53-78.

VYGOTSKI, L. (1935/85 tr. fr.) : Pensée et langage. Paris, Messidor (Terrains).

WERTSCH, J. V. (1985): Culture, Communication and Cognition, Vytgotskian perspectives. Cambridge, Cambridge University Press.

WERTSCH, J. V. (1985) : "A sociocultural approach to socially shared cognition", in L. B. RESNICK, J. M. LEVINE \& S. D. TEASLEY (eds.) : Perspectives on socially shared cognition. American psychological Association. Washington, 85-100.

WONG-FILLMORE, L. (1976) : The Second Time Around: Cognitive and Social Strategies in Second Language Acquisition. Ph. D. dissertation, Stanford University.

WONG-FILLMORE, L.(1989) : «Language learning in social context : the view from research in second language learning », in R. DIETRICH \& F. GRAUMANN (eds.) : Language processing in social context, Amsterdam Elsevier, 277-302. 